\title{
A NOISE-TOLERANT EVOLUTIONARY ALGORITHM BASED ON ACCUMULATIVE SAMPLING
}

\author{
Jeongmin Kim, Steven Kurniawan and Kwang Ryel Ryu \\ Department of Electrical and Computer Engineering, Pusan National University \\ 2, Busandaehak-ro 63beon-gil, Geumjeong-gu, Busan, 46241, Republic of Korea
}

\begin{abstract}
This paper proposes an evolutionary algorithm that can efficiently solve noisy optimization problems for which the objective functions can be evaluated not accurately but only approximately. While the accuracy of evaluation may be improved by taking many samples of evaluation and taking the average, it can be computationally demanding. The proposed algorithm is an improvement over a previous work to achieve higher search efficiency by better allotting different number of samples to different candidate solutions in the population during the course of evolution. Experiments with a set of benchmark problems show that the proposed algorithm significantly outperforms the previous algorithm.
\end{abstract}

\section{KEYWORDS}

Noisy Optimization, Evolutionary Algorithm, Restricted Tournament Selection

\section{INTRODUCTION}

In this paper, we apply the idea of accumulative sampling (Park and Ryu, 2011) to solving noisy optimization problems by using an evolutionary algorithm called the restricted tournament selection (RTS) (Harik, 1995). Many real-world optimization problems are noisy in the sense that their objective functions cannot easily be evaluated accurately. A good example of such kinds can be seen when we have to conduct many simulations to obtain an accurate evaluation of a candidate solution. If we make the simulation simpler by reducing the simulation scenarios in an attempt to save the computation time, the resulting evaluation becomes inaccurate or noisy. A baseline approach to solving a noisy optimization problem would be to use what is called a simple resampling method that takes a large number of samples of fitness (i.e., objective evaluation) for every candidate solution to reduce the effect of noise. However, such an approach makes sense only for the problems that does not demand much computation time in obtaining each fitness sample. The accumulative sampling (AS), which can be considered as a kind of dynamic resampling, allots different number of samples to different candidate solutions during the course of evolution under the framework of an overlapping-population model or a generational model equipped with an archive. AS gives only a minimum number of samples when a solution is first generated, but then allots more samples to those that survive longer in the archive (Park and Ryu, 2011) or in the overlapped population (Jang et al., 2012), and updates the fitness by using both newly and previously sampled values to obtain a more reliable estimate. As the selection between the competitors is made probabilistically, estimates form larger samples result in more reliable decisions. Given a fixed number of total evaluations, using AS has been shown to search more efficiently under noisy environment by better utilizing the computational resources for evaluation.

The noisy optimization algorithm proposed in this paper is an extension of the algorithm called NTGA proposed by a previous work (Jang et al., 2012), which is basically an AS method implemented on top of Harik's RTS. The main difference between our algorithm and NTGA lies in the way additional samples are given to the individuals in the population. While NTGA unconditionally gives an additional sample to an individual whenever it becomes a competitor for survival selection against a newly born, our algorithm does so only when the result of probabilistic comparison between the two is not decisive. By allotting additional samples more conservatively, our algorithm makes use of the computational resources more efficiently and thus achieves better search performance for optimization under uncertain environment. Experiments with some benchmark functions show that the proposed algorithm outperforms NTGA significantly. 


\section{PROPOSED ALGORITHM}

Figure 1 shows the pseudocode of our proposed algorithm named NT-RTS (noise-tolerant RTS). As is done in the original RTS, $m$ offspring are generated from $m$ random parents without reference to their fitness. Each offspring $y$ is evaluated by sampling $n_{i}$ times and then probabilistically compared with a competitor for a survival selection. As the competitor, $t$ individuals are randomly selected from the population and then the one most similar to $y$ is selected. Notice that the competitor $x$ already has some fitness samples accumulated to it. If their average looks better or worse than that of $y$ with a high confidence, the winner is decided right away. Otherwise, the comparison is done again after $n_{a}$ additional samples are given to $x$ to revise its evaluation. Then, the final winner (either $x$ or $y$ ) replaces $x$ in the population.

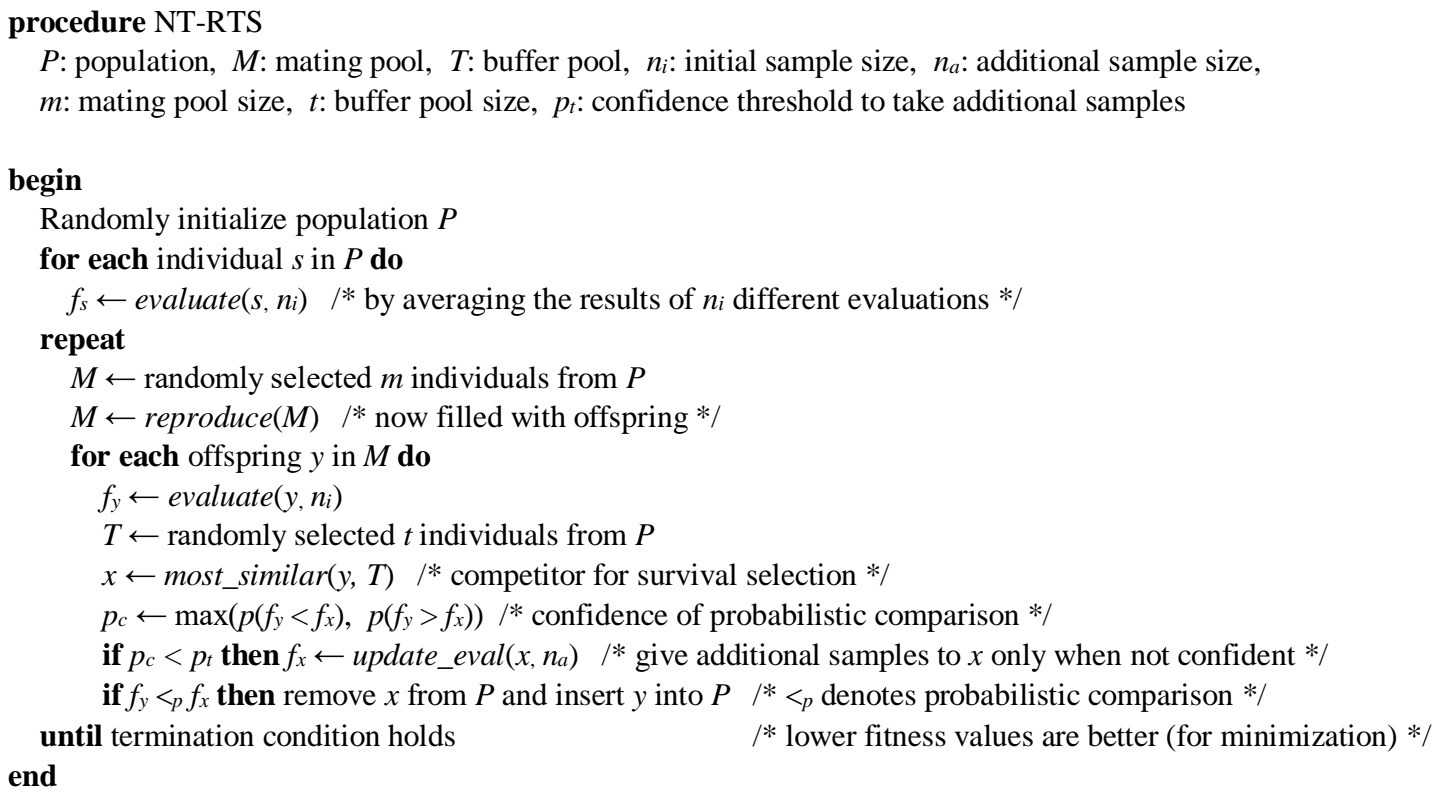

Figure 1. Pseudocode of NT-RTS

\section{RESULTS AND FUTURE WORKS}

NT-RTS has been compared with the simple resampling method and NTGA using three benchmark functions: Ackley, Dropwave, and Alpine (Jang et al., 2012). The dimension of the functions are set to 30 and additive zero-mean Gaussian noise is added to make the problem noisy. The population size is set to 100, and the total number of evaluations (i.e., samples) is fixed to 100,000. Other parameter settings are $n_{i}=2, n_{a}$ $=1, m=2, t=50$, and $p_{t}=0.99$. The resampling method is set to constantly give 5 samples to each individual. Table 1 shows the results of paired $t$-test after running each case 50 times. More investigation shows that NT-RTS evaluates 5 6\% more candidate solutions than NTGA by making an efficient use of the allowed 100,000 limit, which seems to be the main reason of the dominant performance by NT-RTS. Our future works include more experiments with more benchmark functions with more careful parameter tuning. We will also apply NT-RTS to some real world problems of simulation-based optimization.

Table 1. Results of paired $t$-test using benchmark functions ('+' indicates that NT-RTS is significantly better)

\begin{tabular}{ccccccccccc}
\hline & \multicolumn{3}{c}{ Ackley } & \multicolumn{3}{c}{ Dropwave } & \multicolumn{3}{c}{ Alpine } \\
\hline Noise level & Low & Medium & High & Low & Medium & High & Low & Medium & High \\
\hline Resampling & + & + & + & + & + & + & + & + & + \\
NTGA & + & + & $=$ & $=$ & + & + & + & + & + \\
\hline
\end{tabular}




\section{ACKNOWLEDGEMENT}

This research was supported by the MSIT (Ministry of Science and ICT), Korea, under the ICT Consilience Creative program (IITP-2019-2016-0-00318) supervised by the IITP (Institute for Information \& communications Technology Planning \& Evaluation).

\section{REFERENCES}

Harik, G. R., 1995. Finding multimodal solutions using restricted tournament selection, Proceedings of the Sixth International Conference on Genetic Algorithms. San Francisco, CA, pp. 24-31.

Jang, H., Choe, R. and Ryu, K. R., 2012. Deriving a robust policy for container stacking using a noise-tolerant genetic algorithm. Proceedings of the 2012 ACM Research in Applied Computation Symposium on - RACS '12. New York, USA, pp. 31-36.

Park, T. and Ryu, K. R., 2011. Accumulative sampling for noisy evolutionary multi-objective optimization. Proceedings of the 13th annual conference on Genetic and evolutionary computation - GECCO '11. Dublin, Ireland, pp. 793-800. 